\title{
LAS SALVEDADES (PROVISOS) Y LA MAGNITUD DEL CAMBIO TEÓRICO*
}

\author{
HERnÁn Miguel \\ Universidad de Buenos Aires \\ herny@mail.retina.ar \\ JORGE PARUELO \\ Universidad de Buenos Aires \\ jparuelo@mail.retina.ar \\ Guillermo Pissinis \\ Universidad de Buenos Aires \\ gpissinis@ciudad.com.ar
}

RESUMEN: Las fallas predictivas obligan a alguna modificación en las teorías, lo cual, en muchos casos, consiste en detectar algún factor que ha interferido en el experimento. Podría ser que se tratara de un factor de un tipo ya reconocido por la teoría o bien que fuera necesario proponer un factor de un tipo no conocido hasta el momento. En este trabajo proponemos un ordenamiento de los cambios en las teorías de acuerdo con el tipo de factor propuesto en la modificación. Este ordenamiento puede utilizarse para distinguir los cambios en ciencia normal de los cambios revolucionarios. Nuestra propuesta se realiza sobre la base de que una teoría admite únicamente un número finito de tipos de factores.

PALABRAS CLAVE: Kuhn, cambio científico, anomalías, racionalidad

SUMMARY: The failure of a prediction forces some modification of the theory, which in many cases means locating some factor that interfered in the experiment. A factor may be found of a type already recognized by the theory. Or it may be the case that it becomes necessary to propose an as yet unknown factor. Here we suggest an ordering of theory changes according to the type of factor proposed. The ordering may be used to distinguish normal change

${ }^{1}$ Este trabajo fue realizado en el marco del proyecto de investigación que dirige Eduardo H. Flichman con subsidio otorgado por UBACyT. Agradecemos a él, a Horacio Abeledo, Martha Clarizza y Susana Carsolio, miembros del equipo de investigación, las sugerencias aportadas durante la discusión de este trabajo. También agradecemos a Víctor Rodríguez y a Ana Rosa Pérez Ransanz las sugerencias realizadas y al profesor Gregorio Klimovsky sus comentarios en ocasión de haber presentado una versión previa de este trabajo. 
from revolutionary change. Our proposal is based on the thesis that a theory admits only a finite number of types of factors.

KEY WORDS: Kuhn, scientific change, anomalies, rationality

\section{Introducción}

El problema del cambio teórico, es decir la búsqueda de patrones racionales de cambio de teorías, marcos conceptuales, paradigmas $\mathrm{u}$ otras estructuras que puedan proponerse para describir el devenir científico, ha sido y es un problema con múltiples aristas. En particular, el tratamiento de este problema en un marco kuhniano es tema de discusión en el ámbito de la filosofía de la ciencia. Se han llevado a cabo diversos abordajes al problema del cambio científico. Ana Rosa Pérez Ransanz presenta un buen panorama de esto a la vez que introduce sus propias propuestas y sugiere posibles líneas de trabajo. ${ }^{1}$ Creemos que los distintos abordajes, desde distintas perspectivas, son producto de lo complejo que resulta el problema del cambio teórico en sí mismo, y que no debe soslayarse ninguna línea en la búsqueda de un marco teórico que dé cuenta del problema. El presente trabajo pretende abordar dicho problema al establecer una tipología de los cambios en relación con los elementos que los impulsaron. ${ }^{2}$

${ }^{1}$ Enfocando el problema del cambio teórico desde una perspectiva semántica, Pérez Ransanz (1999, pp. 93-94) dice: "la mayoría de los cambios de significado, aquellos que ocurren en los periodos de ciencia normal, no implican alteraciones en la forma de agrupar los objetos, ni por lo tanto en la estructura del ámbito de referencia [...]. No todo desarrollo semántico implica cambios en la estructura conceptual vigente, ni por tanto genera inconmensurabilidad." Pero este tipo de cambio de significado sí podría acarrear nuevas relaciones entre tipos de objetos ya conocidos en la teoría, con el correspondiente cambio en el marco conceptual. Esta línea de investigación, en la que aparece un cambio teórico que puede resultar significativo sin que exista inconmensurabilidad, está fuera de los objetivos de este trabajo, pero es un camino que merece explorarse.

${ }^{2}$ Aunque intentamos describir los distintos tipos de procedimientos lógicos y metodológicos que subyacen al cambio teórico, no abordaremos el problema de en qué condiciones están dispuestos los científicos a revisar sus creencias a la luz de la evidencia disponible. El proceso de revisión de creencias exige consideraciones que exceden los límites de este trabajo. Siguiendo el modelo de revisión de creencias propuesto por P. Gärdenfors (1988) nos hemos ocupa- 
Con este fin se utilizará el concepto de proviso (salvedad) que fue desarrollado por C.G. Hempel (1988). A partir de este concepto y adoptando en este análisis una perspectiva cercana a la de Kuhn, caracterizamos enigma y anomalía para establecer una gradación de cambio teórico, con el fin de disponer de una herramienta útil para la búsqueda de patrones que permitan una tipología de los cambios, al menos en una importante cantidad de casos.

En lo que sigue describimos el concepto de proviso propuesto por Hempel, a partir del cual desarrollamos nuestra caracterización de enigma y anomalía para luego intentar establecer la tipología del cambio teórico.

\section{Las salvedades y el carácter inferencial de las teorías}

Desde muy diversos puntos de vista se ha insistido en que una de las facetas más importantes de una teoría científica es su carácter inferencial. Junto a la explicación de los fenómenos empíricos, las teorías científicas nos proporcionan un procedimiento inferencial que nos lleva de ciertos enunciados, llamados hipótesis y condiciones iniciales, a otros enunciados que describen una situación aún no observada. Seamos realistas o instrumentalistas en nuestra interpretación de los enunciados de una teoría, la capacidad deductiva que ésta tiene es un aspecto relevante a la hora de brindarnos una explicación de los fenómenos observados o de posibilitarnos su contrastación por medio de las predicciones que derivamos. Así, estimamos que dada la masa de un cuerpo, su posición y velocidad en un tiempo $t_{1}$ y las fuerzas que lo afectan durante el lapso entre $t_{1}$ y $t_{2}$, es posible deducir, en virtud de las leyes de la mecánica, su posición en un tiempo $t_{2}$. La teoría mecánica de Newton funciona así como un mecanismo inferencial que nos permite hacer predicciones exitosas partiendo del conocimiento de ciertos datos iniciales. Ahora bien, esta función inferencial de las teorías científicas queda seriamente comprometida, según hace notar Hempel (1988), por

do de este problema en dos trabajos anteriores. Véase H. Miguel, J. Paruelo, y G. Pissinis $(1999,2000)$. 
la presencia de ciertas cláusulas ineludibles llamadas "provisos" (salvedades).

¿Qué son estas cláusulas adicionales? Las salvedades son presuposiciones no explícitas de la inferencia teórica, que están presentes cuando queremos, por ejemplo, deducir la afirmación empírica "si la barra $b$ se quiebra en dos barras y ambas quedan suspendidas una cerca de la otra y a la misma distancia del suelo, entonces se orientarán formando una línea recta" tomando como punto de partida los enunciados teóricos "la barra $b$ es un imán" y "si un imán se quiebra en dos barras, entonces ambas barras son imanes y sus polos se atraen o se repelen". Por cierto que podemos aceptar esto último junto a las demás leyes del magnetismo y sin embargo cabe la posibilidad de que ambas barras no se orienten en la dirección predicha debido a la existencia de un campo magnético que modifica la posición final de las barras, o bien de algún otro elemento perturbador, por ejemplo, una fuerte corriente de aire, que altera el resultado predicho. Ya que las leyes del magnetismo no garantizan la ausencia de tales fuerzas, diremos que el enunciado observacional no se deduce simplemente de la hipótesis, sino de ésta más la suposición adicional de que las barras suspendidas no están sometidas a ninguna influencia perturbadora.

Estas suposiciones funcionan como premisas ineludibles para poder deducir la predicción. Sin ellas, el pasaje de la teoría a la observación no es una deducción, puesto que la teoría puede ser verdadera y sin embargo ser falsa la predicción cuando no se cumplen las salvedades. Incluso se necesitan salvedades para las inferencias deductivas entre las mismas leyes de la teoría. Así, del enunciado "la barra $b$ es un imán" no se deduce que "si un imán se quiebra en dos barras, $a$ y $b$, entonces ambas barras son imanes y sus polos se atraen o se repelen", puesto que si rompemos la barra a una temperatura muy elevada, las barras podrían desmagnetizarse.

¿Qué afirma una salvedad? La salvedad presupuesta para hacer una inferencia teórica afirma que en un caso dado, como el de las barras magnéticas, no está presente ningún otro factor relevante excepto aquellos especificados en las condiciones ini- 
ciales y de contorno (de ahora en adelante $S_{1}$ ). Así, los factores a los que se refiere la salvedad son aquellos que, de estar presentes, modificarían el resultado final. Por eso la salvedad constituye una presunción de completitud, pero no de completitud epistémica, sino óntica, pues no sólo exige que $S_{1}$ exprese toda la información disponible y relevante acerca de la ocurrencia de un hecho, sino que además presupone que no existen en la realidad otros factores que afecten el resultado de la predicción más que los especificados en $S_{1}$. (De aquí en adelante llamare$\operatorname{mos} S_{2}$ al enunciado correspondiente a la predicción.) Lo que afirma la salvedad entonces es que los factores citados en $S_{1}$ son todos los que forman el conjunto de hechos "nómicamente relevantes" para que se cumpla lo dicho en $S_{2}$ si la teoría funciona correctamente.

\section{La predicción y el papel de las salvedades}

Se comprende que cualquier predicción que permita poner a prueba la teoría contendrá entre sus premisas una afirmación acerca de la ausencia de factores intervinientes que pudieran hacer fracasar tal predicción. Pero esta afirmación es, como recién dijimos, de carácter óntico ya que no solamente se refiere a los factores reconocidos por la teoría en cuestión como posibles perturbadores del resultado final, sino que además incluye la no ocurrencia de cualquier otro factor tanto de los que estamos dispuestos a aceptar, como de aquellos de cuya existencia ni siquiera hemos sospechado. Esto último será de gran relevancia a la hora de explicar el fracaso de una predicción.

Un razonamiento predictivo se compone, entonces, de un enunciado que describe una situación empírica inicial, las leyes de la teoría más las hipótesis auxiliares explícitas y, por último, la suposición acerca de la ausencia de factores perturbadores intervinientes. De ellos se deducirá una situación empírica final o predicción. 
Esquemáticamente:

1. $\mathrm{S}_{1}$ (situación empírica inicial) ${ }^{3}$

2. Leyes de la teoría

3. Hipótesis auxiliares explícitas

4. No ocurren factores perturbadores del resultado

\section{$\mathrm{S}_{2}$ (situación empírica final o predicción)}

El cuarto enunciado - el que menciona la ausencia de factores perturbadores - es una salvedad cuyo contenido trataremos de aclarar un poco más. Podemos clasificar los factores relevantes capaces de hacer fracasar la predicción en tipos de factores intervinientes. Así, en el ejemplo de las barras de metal que cuelgan de un hilo, y que de acuerdo con nuestra teoría son imanes, afirmamos que se alinearán siempre y cuando no haya corrientes de aire, otros campos magnéticos, otras fuerzas elásticas, etc. Esta enumeración pone de manifiesto cierta serie de tipos de factores: la serie de los tipos de factores ya conocidos en virtud de lo que afirman nuestras teorías y nuestros compromisos ontológicos vigentes; $\mathrm{o}$, dicho de otro modo, todo aquello que dentro del marco conceptual vigente, o el paradigma, se acepta como los tipos de entidades y procesos que constituyen el mundo. Llamemos a estos tipos de factores $F_{1}, F_{2}, F_{3}, \ldots F_{n}$, donde cada subíndice alude a un tipo distinto de factor.

Ahora bien, debido a que la salvedad es una afirmación de carácter óntico que excede el alcance epistémico de las afirmaciones que estamos dispuestos a admitir según nuestras teorías vigentes, ha de referirse también a tipos de factores desconocidos. Llamémoslos $D_{1}, D_{2}, D_{3}, \ldots$, donde cada subíndice alude a un tipo distinto de factor. En principio aceptamos que estos tipos de factores pueden ser infinitos ya que no parece conveniente recortar de antemano la ontología. En el caso de los tipos de factores conocidos, los $F_{i}$ mencionados antes, está claro que

${ }^{3}$ Estrictamente hablando, nos referimos al enunciado que corresponde a la situación empírica inicial, y análogamente se obtiene en la deducción un enunciado que corresponde a la situación empírica predicha. 
no pueden ser infinitos, ${ }^{4}$ aunque sí lo puedan ser los factores, es decir los valores que toma cada tipo de factor. El tipo de factor "corriente de aire" puede tomar valores de $30 \mathrm{~km} / \mathrm{h} \mathrm{o} 100 \mathrm{~km} / \mathrm{h}$, etc., éstos son algunos de los valores que puede tomar el tipo de factor corriente de aire, es decir, alguno de los factores que pueden modificar la predicción.

Nuestro esquema de predicción puede reescribirse del siguiente modo:

1. $S_{1}$ (situación empírica inicial)

2. Leyes de la teoría

3. Hipótesis auxiliares explícitas

4. No ocurren factores de tipo $F_{1}, F_{2}, \ldots F_{n}$ ni ocurren factores de tipo desconocido $\left(D_{1}, D_{2}, D_{3}, \ldots\right)$ que perturben el resultado

\section{$\mathrm{S}_{2}$ (situación empírica final o predicción)}

Recordemos que, de ocurrir uno u otro tipo de factor, la predicción fracasaría ya que $F$ y $D$ constituyen una descripción exhaustiva de los factores relevantes. Está claro que tal fracaso no será entendido directamente como una refutación de la teoría; aunque si la teoría es incorrecta, tal vez sea ella la responsable de la falla predictiva. Pero, en principio, el proceso de revisión puede seguir algunas de las siguientes alternativas:

1. Revisar si se cumplen las condiciones iniciales.

2. Rechazar la teoría.

3. Revisar el cumplimiento de las hipótesis auxiliares explícitas.

4. Investigar cuál de los factores de tipo $F_{1}, F_{2}, F_{3}, \ldots F_{n}$ ha ocurrido.

${ }^{4}$ Los tipos de factores conocidos tienen que haber sido mencionados y eventualmente caracterizados por algún científico en alguna ocasión. Si tenemos en cuenta que el conjunto formado por todo lo dicho por algún ser humano desde que se desarrolló la primera forma de lenguaje contiene un número finito, aunque inmensamente grande, de frases, entonces los dichos de los científicos también son un número finito, ya que son un subconjunto de las anteriores. 
Es obvio que no pueden revisarse los factores de la serie de tipos $D$, pues, según las teorías vigentes, éstos no existen. Así lo demuestra Hempel cuando dice que en la búsqueda de las influencias perturbadoras durante una etapa de ciencia normal se considerarán solamente los tipos de factores reconocidos por una u otra de las teorías científicamente aceptadas.

Las revisiones 1 y 3 nos parecen obviamente viables. Verificar que se cumplan las condiciones iniciales solamente involucra la dificultad de la carga teórica de la observación. Además, la revisión 3 se refiere a verificar condiciones de contorno, algunas condiciones iniciales auxiliares para el caso en cuestión y quizás a la puesta a prueba de leyes o teorías auxiliares.

Ahora ocupémonos de la revisión 4. Se deben investigar los factores de la serie de tipos $F$ que según nuestras teorías aceptadas son relevantes en la deducción de la predicción. Analicemos cuántos factores debemos revisar.

Como ya dijimos, no es posible que nuestra descripción del mundo, es decir el compromiso ontológico que se desprende de nuestro paradigma, incluya una cantidad infinita de tipos de factores. Puede haber combinaciones de factores, pero éstas serán un tipo de factor que es combinación de un número finito de tipos y por lo tanto también sumarán un número finito. ${ }^{5}$ Esto es que la predicción puede fallar porque ha estado presente un factor de tipo $F_{1}$, o bien uno de tipo $F_{2}$, o bien de $F_{3}, \ldots$ o bien de $F_{n} ;$ y también puede fallar porque ha estado presente la combinación de factores de tipo $F_{1}$ y $F_{2}$, o bien la combinación de $F_{1}$ con $F_{3}$, etc. ${ }^{6}$

Cierto es, en cambio, que cada uno de los tipos de factores (corriente de aire, campo magnético externo, fuerzas elásticas, etc.) se refiere a una cantidad infinita de perturbaciones. Por ejemplo, cuando decimos que no existe una corriente de aire que perturbe el experimento, esto indica que no está presente

${ }^{5}$ El número de combinaciones posibles puede ser muy grande, pero siempre finito. El número de tipos combinados es el número que surge de considerar la suma de todas las combinaciones posibles de los tipos conocidos, digamos $n$, tomados de a uno, dos, tres, hasta $n$, sin repetición de tipos.

${ }^{6}$ Agradecemos a H. Abeledo una serie de consideraciones que nos ayudó a fijar esta posición. 
una corriente de aire de intensidad 1 , ni una de intensidad 2, ni una de intensidad entre 1 y 2 que es un rango infinito y no numerable. Así, cada tipo se refiere a una cantidad infinita de factores. Podemos expresar esto brevemente agregando al símbolo del tipo de factor una variable $x$ que toma sus valores de cierto conjunto de números que puede ser, y en muchos casos lo es, infinito y no numerable. Así, para el tipo $F_{1}$ tendremos una colección infinita de factores $F_{1}(x)$; para el tipo $F_{2}$, otra colección $F_{2}(x)$; y así para cada tipo de la serie de los tipos de factores conocidos.

Entonces, ¿cómo podremos asegurar que no intervienen factores de tipo $F_{1}$, por ejemplo, si debemos revisar infinitos factores $F_{1}(x)$ ?

La respuesta a este problema epistemológico debemos buscarla, paradójicamente, en la forma en que los científicos operan experimentalmente. Los aparatos de medición detectan el valor $F_{i}(x)$ para el tipo específico de factor $F_{i}$. Así, siguiendo con nuestro ejemplo, un detector de corrientes de aire marcará la intensidad de la corriente existente en ese lugar y para ello recorrerá un rango infinito de valores de $F_{1}(x) .{ }^{7}$ También es cierto que el detector tendrá un valor mínimo por debajo del cual no indica la presencia de corrientes de aire aunque las hubiera. En ese caso existe otro rango infinito de valores $F_{1}(x)$ que el detector no registra. Pero he aquí que la aventura científica nos ha llevado a diseñar un detector cuyo valor mínimo de registro sea menor que la intensidad capaz de perturbar el experimento de modo que nuestro detector registra cualquier valor $F_{1}(x)$ nómicamente relevante. ${ }^{8}$ Es cierto también que todo detector tiene un límite superior de registro; por ejemplo, nuestro detector no sirve para registrar un huracán, ya que fue diseñado para detectar corrientes de aire de baja intensidad — aunque también está claro que se rompería. Es decir, no puede registrar el valor de la intensidad del viento, pero puede detectar la presencia

${ }^{7}$ Asumiendo que $F_{1}$ es el tipo de factor "corriente de aire".

${ }^{8}$ El umbral de un instrumento se asocia con su sensibilidad, y esto es crucial en la detección de la presencia de determinado factor. En cambio, al momento de querer determinar el valor $F_{i}(x)$, la característica de interés será la precisión. 
de un factor perturbador interviniente de intensidad mayor que la de su escala de medida. ${ }^{9}$ Sigue entonces siendo útil para decidir sobre la existencia de factores $F_{1}(x)$. Por lo tanto, necesitamos un detector de estas características, que por otro lado son las habituales, por cada tipo de factor que creemos, según nuestras teorías vigentes, que puede perturbar el resultado del experimento. Y esto es exactamente lo que hacen los científicos defensores de la teoría cuando se encuentran con un fracaso predictivo.

Ya no es un problema que el rango de los factores de un mismo tipo sea infinito. Resta preocuparse de las combinaciones de factores. Esto se resuelve mucho más fácilmente ya que un detector diseñado para registrar los factores de tipo $F_{1}$ los detectará aun cuando esté presente otro factor de tipo $F_{2}$, siempre y cuando este segundo tipo no interfiera con el primero a modo de neutralizarlo o disminuirlo. Si esta interacción negativa ocurriera y el detector no registrara el primer factor por estar "disimulado" por el segundo, entonces nuestro arreglo experimental tampoco lo detectará y la predicción no fallará. En síntesis, lo que perturba al experimento también perturba al detector.

En consecuencia, la tarea de la revisión se reduce a diseñar un detector ${ }^{10}$ para cada tipo de factor $F_{1}, F_{2}, F_{3}, \ldots F_{n}$. Una vez revisados todos los tipos de factores que nuestras teorías y compromisos ontológicos sostienen que existen, estaremos en condiciones de sostener que: o bien la teoría se enfrenta a un

${ }^{9}$ También podría ocurrir que el factor interviniente ni siquiera influyera sobre el medidor. Por ejemplo, un sonido de mayor frecuencia de la que puede excitar al detector y que tampoco lo daña. Para estos casos sostenemos que tampoco este tipo de señal es perturbadora, de modo que sigue siendo válido que el detector cumple la función de denunciar los factores de interés para iniciar el proceso de revisión. En caso de que esa señal fuera perturbadora pero no fuera registrada por ese detector, se seguirá que es necesario diseñar un detector específico para tales fines como si fuera para otro tipo de factor más. En todo caso, para cada tipo de factor relevante se diseñará un detector específico en el rango en que tales factores puedan perturbar el resultado del experimento.

${ }^{10} \mathrm{O}$ un conjunto de detectores de manera tal que se cubra el rango de valores que resulta nómicamente relevante para el suceso predicho. 
posible caso de falsación, ${ }^{11}$ o bien existen otros factores que no conocemos, que pueden estar interviniendo para dar cuenta del fracaso predictivo. Es importante notar que estos factores no son de los tipos aceptados por nuestras opiniones ontológicas vigentes.

Resulta pues razonable que, a la luz de las teorías vigentes y de los compromisos ontológicos correspondientes, el científico comience por revisar las salvedades que aluden a los factores de tipo $F$. Coincidimos con Hempel cuando señala que la búsqueda de influencias perturbadoras durante una etapa de ciencia normal considerará solamente los tipos de factores reconocidos por las teorías como nómicamente relevantes. ${ }^{12}$ Pero Hempel se limita a decir que, en el caso de una persistente falla en encontrar entre dichos factores el causante de la perturbación, se podría producir un cambio del marco conceptual. Así se obtendría una explicación de esos fracasos sin tener que recurrir a las salvedades que a la luz de este nuevo marco hablaban de cosas inexistentes. ${ }^{13}$

Por nuestra parte creemos que, teniendo en cuenta la distinción entre factores $F$ y $D$, el análisis de la conducta de los científicos durante la ciencia normal y durante las revoluciones científicas adquiere un nuevo matiz que nos permite avanzar en la explicación de la naturaleza del cambio científico.

\section{Las salvedades en la ciencia normal: la naturaleza de los enigmas y las anomalías}

Veamos de qué manera podemos interpretar las ideas de Kuhn a la luz de la distinción de factores hecha anteriormente.

Sabemos que, para Kuhn, los problemas que tiene que enfrentar un científico son de dos tipos diferentes: o un enigma o una anomalía. Un enigma es un problema que tiene solución

\footnotetext{
${ }^{11}$ Un caso interesante de este tipo es aquel en el cual hay un factor de tipo conocido que se encuentra que es relevante para la predicción llevada a cabo pero que previamente al fracaso predictivo no era considerado nómicamente relevante. Esto llevará a la modificación de las leyes de la teoría.

${ }^{12}$ Véase Hempel 1988, p. 33.

${ }^{13}$ Hempel 1988, p. 33.
} 
dentro del paradigma; es decir, un problema cuya solución no implica un cambio revolucionario. Una anomalía es un problema que se resiste a ser resuelto, o bien uno cuya resolución (o disolución) implica un cambio revolucionario y, por lo tanto, un cambio de paradigma. Además sabemos que, para Kuhn, esa "matriz disciplinaria" que cuenta con "concretas soluciones de problemas" puede considerarse un paradigma. ${ }^{14}$ Pero si prestamos atención a los ejemplos de resolución de enigmas que el mismo Kuhn nos da, veremos que se hace necesario caracterizar de un modo más preciso en qué consiste el trabajo del científico al resolver esos rompecabezas.

Ejemplos de enigmas que no producen novedades inesperadas en el paradigma vigente son los referidos a la resolución de problemas tales como "la posición y magnitud de las estrellas, los periodos de eclipses binarios de los planetas"; $;^{15}$ el desarrollo de instrumental adecuado para la confrontación de las predicciones de la teoría; ${ }^{16}$ la determinación de constantes físicas, la obtención de leyes cuantitativas (como la Ley de Boyle o la fórmula de Joule que relaciona el calor generado con la resistencia eléctrica): ${ }^{17}$ la medición de una longitud de onda, los resultados del calentamiento por comprensión, etc. ${ }^{18}$ Cada problema en la categoría de enigma puede ser visto "como un problema [...] ya $[\ldots]$ encontrado antes". 19

Un segundo tipo de enigmas está relacionado con el desafío de encontrar las condiciones y factores intervinientes para explicar una falla predictiva sin modificar las leyes de la teoría.

${ }^{14}$ Esta manera de caracterizar un paradigma se encuentra en la postdata de 1969, en Kuhn 1969, pp. 280-286. Coincidimos además con Putnam (1974, p. 229) cuando sostiene que la manera menos ambigua de ver un paradigma es tomarlo como una teoría que cuenta con un ejemplo impactante de aplicación exitosa.

${ }^{15}$ Kuhn 1962, p. 54.

${ }^{16}$ Kuhn 1962, pp. 55-56.

${ }^{17}$ Kuhn 1962, p. 58.

${ }^{18}$ Kuhn 1962, p. 68.

${ }^{19}$ En Kuhn (1969, p. 290) refiriéndose a los estudiantes de ciencias. Las cursivas son del autor. 
Antes de considerar con más detalle este tipo de enigma, nos parece útil tener presente cómo esquematiza Putnam (1974) los distintos tipos de problemas científicos para poder comprender mejor las diferencias que pretendemos trazar en nuestro trabajo. Los esquemas son los siguientes:

\begin{tabular}{ll} 
Esquema I & Teoría \\
& Afirmaciones auxiliares \\
\cline { 2 - 2 } Esquema II & Teoría \\
& ???????? \\
\cline { 2 - 2 } & $\begin{array}{l}\text { Explanandum (enunciado del hecho } \\
\text { que va a ser explicado) }\end{array}$
\end{tabular}

Esquema III Teoría

Afirmaciones auxiliares

Consecuencia????????????

El primer esquema se ocupa del clásico problema de la justificación racional del cambio teórico, visto por el lado de la confirmación inductivista o desde la perspectiva del falsacionismo: se trata de saber si las predicciones hechas por la teoría se cumplen o no para decidir si seguimos usándola o la cambiamos. El segundo esquema tiene que ver con hallar aquellos supuestos que en conjunción con la teoría llevarían a explicar satisfactoriamente un hecho ocurrido. El tercer esquema tiene que ver con un problema de índole más bien matemática o inferencial: ${ }^{20}$ el de saber qué consecuencias pueden sacarse de la teoría en conjunción con sus supuestos adicionales.

El esquema II nos muestra cómo se plantea el enigma. Tal como lo viene diciendo Kuhn, éste aparece al querer aplicar el paradigma a una situación nueva. Lo que no se sabe es cómo

${ }^{20}$ Putnam (1974) sostiene que este tipo de problemas fue dejado de lado por ser problemas "puramente matemáticos" (p. 231). 
ha de compaginarse la teoría con el hecho que va a ser explicado. Resolver esta clase de enigmas es, entonces, encontrar las afirmaciones auxiliares que permitan aplicar la teoría para explicar ese hecho y así ponerla a prueba exitosamente. Pero estos casos de resolución no llevan a ninguna novedad, sólo son un ejemplo de exitosa compaginación de la matriz disciplinaria con las situaciones empíricas.

Sin embargo, como dijimos anteriormente, existe una segunda clase de enigmas, la que se le presenta al científico cuando está frente a una falla predictiva; por ejemplo, cuando se observó que la órbita de Urano no coincidía con la predicha por la teoría. En casos como éste, el científico en ciencia normal propondrá que hay algún factor perturbador que explica el resultado observacional. Para esto no bastará con echar mano de los recursos heurísticos provistos por la teoría, sino que tendrá que postular, además, algo novedoso para solucionar el enigma en cuestión, que en el caso de este ejemplo fue proponer la existencia de un octavo planeta. ${ }^{21}$

La resolución consiste en estos casos en conjeturar como hipótesis auxiliar la intervención de algún factor perturbador de modo que, si este factor no hubiera estado presente, la teoría habría tenido éxito en la predicción. Habrá que determinar el tipo de factor $F$ ("planeta" en el ejemplo), y las características específicas del planeta correspondiente (masa, ubicación, etc.), para así dar cuenta del problema. En este tipo de respuestas se impone poner a prueba la solución, lo que para los enigmas del primer tipo no era necesario, ya que se trataba simplemente de resolver un caso de aplicación, parecido a lo que todo estudiante debe hacer cuando es adiestrado en el aprendizaje de una disciplina.

Los esquemas II y III nos resultan útiles ahora para ver cómo se desenvuelve la investigación científica. El problema matemático-inferencial podría ser visto a la luz del esquema III como la derivación de las consecuencias observacionales implicadas después de haber agregado las afirmaciones auxiliares. La

${ }^{21}$ Para ampliar este punto puede consultarse H. Miguel, J. Paruelo y G. Pissinis 1997. 
tarea de Leverrier y Adams ${ }^{22}$ fue precisamente ésta: anticipar dónde habría de encontrarse ese octavo planeta y cuáles deberían ser sus características. Esta tarea es una parte esencial de la resolución del enigma. El esquema II no basta para dar cuenta de todo lo que hace un científico en la etapa de ciencia normal. Tras conjeturar la existencia de ese factor (Neptuno, que queda incluido en el tipo de factor planeta, y que a su vez es un factor de la serie de los $F$, es decir, conocido), deberá encontrarse evidencia adicional de que dicho factor estuvo presente. Una vez encontrada esta evidencia ${ }^{23}$ (es decir si verificamos la predicción de acuerdo con lo que dice el esquema I), entonces el enigma habrá quedado resuelto. Ahora podemos agregar en el esquema deductivo para obtener la posición de Urano: "Existe Neptuno con determinada masa, ubicación y velocidad en la órbita $O . "$

¿Qué ocurriría en caso de que continuara la falla predictiva? La falla predictiva podría llevar a seguir insistiendo en la búsqueda de algún otro factor de la serie de tipos $F$ en la medida en que el científico todavía confíe en el paradigma. Pero supongamos ahora que los científicos no pueden encontrar evidencia adicional sobre la ocurrencia de ése u otro factor de la serie de tipos $F$ para explicar el fracaso predictivo. Si, como dijimos antes, tenemos en cuenta que existe la posibilidad, en principio, de realizar una revisión exhaustiva de tales factores, aprovechando que esta serie está compuesta de un número finito de tipos, entonces el problema irá adquiriendo paulatinamente el mote de "anomalía".

Desde nuestra perspectiva, este tipo de problemas no tiene solución dentro del paradigma, debido a que los tipos de factores aceptados en él no dan cuenta del fenómeno.

En la práctica científica no se persigue la exhaustividad de la revisión de los factores conocidos. Los científicos proponen factores desconocidos que puedan dar cuenta de la falla predictiva aun sin haber verificado la totalidad de los factores aceptados por sus teorías. Sin embargo, queremos destacar aquí un proce-

${ }^{22}$ A quienes se les asigna el mérito del descubrimiento de Neptuno.

${ }^{23}$ Dejando de lado la cuestión de cuánta evidencia será necesaria para aceptar que ése fue el factor interviniente. 
dimiento que en principio daría una plataforma de racionalidad para la propuesta de factores desconocidos. ${ }^{24}$ Una vez descartados (en principio) como perturbadores los factores conocidos, los científicos podrían empezar a proponer soluciones externas al paradigma y, en particular, podrían proponer la existencia de factores inadmisibles hasta el momento. Recuérdese que para Kuhn las creencias metafísicas compartidas por los científicos es otro de los componentes de un paradigma. ${ }^{25}$ Entonces, si los factores de la serie de tipos $F$ no alcanzan a solucionar el enigma, el factor propuesto y finalmente aceptado tendrá que ser de la serie de los tipos $D$ (se refiere a tipos de entidades hasta el momento desconocidas y por lo tanto inexistentes en la ontología del paradigma). Ahora, la falla en la predicción será tomada como una anomalía, un problema que no tiene solución en términos de las entidades y procesos aceptados corrientemente.

Podemos ahora caracterizar enigma y anomalía en función de nuestra tesis.

Un enigma es aquel problema para el cual existen factores de la serie de los tipos $F$ tales que, una vez identificados, den cuenta de tal problema.

En el caso de una anomalía, se está ante un problema que se resiste a ser resuelto. Esta resistencia obedece a la inexistencia de un factor de la serie de los $F$ responsable de la falla predictiva

${ }^{24}$ Kitcher (1993, p. 256) propone que los cambios en las prácticas científicas individuales pueden entenderse de acuerdo con lo que él llama "escape tree" y que consiste en un análisis de las pérdidas, compensaciones y modificaciones que se siguen de elegir diferentes caminos al reemplazar, de a uno a la vez, cada miembro del conjunto de enunciados que se ha tornado inconsistente debido a la falla predictiva. En este marco, una de las condiciones para que los científicos consideren la necesidad de cambiar las restricciones típicas de sus prácticas científicas es que cada camino haya quedado bloqueado. Así presentada la propuesta, supone exhaustividad en la revisión de las ramas. Sin embargo, el bloqueo de cada rama está sujeto a la decisión de los científicos ("If the scientists can think of no way of continuing then the path is blocked") y no a la estructura del conjunto de enunciados considerado. De este modo, nuestra exhaustividad a tenor de las teorías vigentes no coincide con la exhaustividad que involucra la decisión de los científicos sostenida por Kitcher.

${ }^{25}$ Véase para esto Kuhn 1969, p. 282. 
y obliga a la aceptación de algún factor de la serie de los $D$ para su solución o disolución.

Obsérvese que estas caracterizaciones no coinciden completamente con las ideas de Kuhn. Si, dado un problema $P$, los científicos, tras sucesivos intentos, no consiguen localizar el factor de la serie de los tipos $F$ responsable del problema, comenzarán a considerar que $P$ es una anomalía. Sin embargo, podría existir dicho factor y simplemente no haber sido localizado, con lo cual $P$ constituiría un enigma para nosotros y una anomalía para Kuhn. ${ }^{26}$

${ }^{26}$ Respecto a la noción de anomalía que puede encontrarse en diferentes pasajes de Kuhn (1962) vale la pena hacer notar (como hicimos con mayor detalle en nuestro (2001)) que en algunos casos las anomalías se asocian con aquellos problemas que no pueden tener solución dentro del periodo de ciencia normal: "no renuncian [los científicos] al paradigma que los ha conducido a la crisis. O sea, a no tratar las anomalías como ejemplos en contrario, aunque, en el vocabulario de la filosofía de la ciencia, eso es precisamente lo que son" (p. 128). Luego del análisis del descubrimiento del oxígeno, agrega que "el valor atribuido a un nuevo fenómeno y, por consiguiente, a su descubridor, varía de acuerdo con nuestro cálculo de la amplitud con la que dicho fenómeno rompía las previsiones inducidas por el paradigma". En estos pasajes, la anomalía queda claramente asociada con la imposibilidad de enmarcarse en las reglas, tipos de soluciones y expectativas del paradigma, y coincidiría plenamente con nuestra caracterización de anomalía. Sin embargo, en el capítulo VIII (p. 134 y ss.), la noción de anomalía parece debilitarse para abarcar tanto los problemas que no tienen solución dentro del marco vigente como aquellos que durante un tiempo no parecieron tener solución, es decir, enigmas de difícil solución. Así, nos dice, refiriéndose al movimiento anticipado del perigeo de la Luna, que (p. 134) "nadie tomó muy en serio esas proposiciones [para modificar la ley del inverso del cuadrado de la distancia] $y$, en la práctica, esa paciencia con una anomalía importante resultó justificada" (las cursivas son nuestras). Y continúa: "Incluso en los casos en que no parece posible que se produzcan errores simples [...] una anomalía reconocida y persistente no siempre provoca una crisis. Nadie puso seriamente en duda la teoría de Newton a causa de las discrepancias, reconocidas desde hacía mucho tiempo, entre las predicciones de esa teoría y las velocidades tanto del sonido como del movimiento de Mercurio. La primera discrepancia fue finalmente resuelta y de manera inesperada, por medio de experimentos sobre el calor, [...] la segunda desapareció al surgir la teoría especial de la relatividad, después de una crisis en cuya creación no había tomado parte. [...] De ello se desprende que para que una anomalía provoque crisis, debe ser algo más que una simple anomalía." Con esto queda claro que hay un uso de la noción de anomalía que tiene que ver con el papel que finalmente cumple en el 


\section{El cambio en ciencia normal y el cambio revolucionario}

Hemos dicho que hay resoluciones de enigmas que no traen aparejado ningún cambio importante ni desde el punto de vista teórico ni desde el punto de vista ontológico. Por supuesto que es legítimo hablar de progreso científico toda vez que hay un problema solucionado. Pero éste es un progreso al estilo de lo que le gustaba al protagonista de Il Gattopardo: cambiar algo para que todo siga igual.

Pero el 'progreso conservador' no es el único tipo de cambio dentro de la ciencia. Resolver un enigma de la segunda clase

cambio revolucionario, más allá de la imposibilidad de solución dentro del marco vigente. Intentando mostrar la dificultad para encontrar una respuesta a cuándo una anomalía interviene en el desencadenamiento de la crisis, agrega (p. 136): "Probablemente hay todavía otras circunstancias que pueden hacer que una anomalía resulte especialmente apremiante $\mathrm{y}$, ordinariamente, se combinarán varias de ellas. Por ejemplo, ya hemos hecho notar que una de las causas de la crisis a que se enfrentó Copérnico fue la sola duración del tiempo durante el que los astrónomos se esforzaron, sin obtener resultados, en reducir las discrepancias residuales del sistema de Ptolomeo. Cuando por esas razones u otras similares, una anomalía llega a parecer algo más que otro enigma más de la ciencia normal, se inicia la transición a la crisis y a la ciencia fuera de lo ordinario." Con estos últimos pasajes vemos que la noción de anomalía está más asociada a su reconocimiento como tal por parte de la comunidad, y esto puede deberse a un efecto de cansancio en la búsqueda de soluciones, más que la inexistencia de soluciones desde el punto de vista de la incompatibilidad del fenómeno con las expectativas del marco vigente, como en cambio se la había caracterizado en el capítulo VI.

Hoyningen-Huene (1993) analiza el problema de la noción de anomalía a lo largo de la obra de Kuhn y aunque menciona parte de esta dificultad, no ofrece una tipificación que aclare esta multiplicidad de usos (p. 225), aunque analiza las condiciones necesarias para el surgimiento de una anomalía que sea tomada como tal. En este sentido, la discrepancia entre cierto fenómeno y las expectativas del marco vigente es una de tales condiciones, pero no es suficiente para la identificación de una anomalía como tal. Vemos que este autor sigue el espíritu kuhniano de rastrear las anomalías que efectivamente desempeñaron un papel en la crisis, aun desde un punto de vista retrospectivo; mientras que nuestro esfuerzo se concentra en la naturaleza de tal discrepancia para detectar el grado de desajuste entre el descubrimiento y las expectativas vigentes.

Más recientemente, Pérez Ransanz (1999) presentó el caso citado anteriormente sobre el desajuste en el perigeo de la Luna como un caso en el que la resolución de la anomalía no involucró un cambio en la teoría (p. 75). 
antes mencionada requiere que se identifiquen los factores que dan cuenta del fallo predictivo, tal como ocurrió con el enigma de la órbita de Urano. Resolver el enigma implicó incluir un factor nuevo de un tipo $F$ de la serie de los tipos de factores conocidos, lo que va acompañado de una novedad: existe una entidad que antes no se conocía, pero cuyo comportamiento cae bajo las leyes del paradigma. Se descubre que existe un particular más de un tipo conocido, y ésta es la novedad ontológica, pero sin cambio teórico.

Distinta es la situación que se le plantea al científico si cree que la solución estaría en algún otro tipo de factor (alguno de la serie de los tipos $D$ ), lo que nos estaría indicando la posibilidad de otra forma de cambio, pues aceptar la existencia de factores de tipos $D$ es aceptar nuevos tipos de entidades y por tanto una modificación en la ontología. Ahora bien, esta modificación puede, a su vez, darse de dos modos diferentes: una modificación que involucre la introducción de un factor de la serie de tipos $D$ compatible con las leyes del paradigma, o bien otra en la que además sea necesario hacer reajustes drásticos en las leyes para describir el comportamiento de esas nuevas entidades.

Un ejemplo del primer modo de modificación (de los últimos dos, que involucran factores de tipo $D$ ) nos lo brinda el caso del descubrimiento del neutrino. Antes de 1930 se conocía la desintegración beta del neutrón: un neutrón emite espontáneamente un electrón ${ }^{27}$ y por tanto da como resultado dos partículas, el electrón emitido y un protón, cuyas cargas eléctricas son opuestas y de la misma magnitud, de manera que se conservaba el valor de la carga eléctrica total anterior a la reacción (que, por tratarse del neutrón, tenía valor nulo). ${ }^{28}$ Según el principio de

\footnotetext{
${ }^{27}$ Para ser rigurosos, la desintegración beta puede consistir en la emisión de un electrón o de un positrón (antielectrón); en ambos casos el número atómico (la cantidad de carga del núcleo atómico) cambia en una unidad.

${ }^{28} \mathrm{La}$ ecuación $n \rightarrow p+e^{-}$debía haber representado este fenómeno radiactivo. Los términos indican que el neutrón en este proceso da como resultado un protón (de carga positiva) y un electrón (de carga negativa). Sin embargo, esta ecuación no representaba correctamente el fenómeno en cuanto al balance de masa-energía como se indica más adelante.
} 
conservación de la energía, otro tanto debía ocurrir con la energía total del sistema y esto era precisamente lo que no ocurría.

Sobre la base de la equivalencia entre masa y energía se supuso que el factor perturbador del resultado experimental se debía a la existencia de una partícula de un tipo enteramente nuevo, con características diferentes de las de las conocidas hasta ese momento: una partícula sin carga eléctrica y de masa muy pequeña con una interacción casi nula con el resto de las partículas. ${ }^{29}$

De hecho tampoco se conservaba el momento angular intrínseco total previamente a postular la existencia del neutrino, ya que tanto el protón como el neutrón y el electrón tienen spin $^{30}$ semientero $(+1 / 2$ o bien $-1 / 2$, medido en unidades en que otras partículas tienen spin igual a la unidad) y por lo tanto la primera ecuación ${ }^{31}$ propuesta $\left(n \rightarrow p+e^{-}\right)$no permite igualar la cantidad total de esta magnitud. Dado lo cual se agrega a la hipótesis de la existencia del neutrino que debe ser una partícula de spin semientero con la cantidad que permita mantener también la conservación de esta magnitud. En pocas palabras, ya que postulábamos la existencia de una partícula de un tipo nuevo, nos podíamos dar todos los lujos y pedirle a esa nueva partícula que tuviera todas las características que necesitabamos para salvar nuestras teorías. Faltaba todavía, como el lector ya imagina, que obtuviéramos evidencias de que esa partícula tenía efectivamente esas características, y, obviamente, esas evidencias no podían provenir del decaimiento beta del neutrón.

Fue Wolfgang Pauli quien poco antes de 1930, mientras estaba dedicado al estudio del decaimiento beta de ciertos elementos

${ }^{29}$ Entonces la ecuación adecuada debía tener en cuenta esta porción faltante, y de ese modo la ecuación de la nota 28 se modifica para dar lugar a la siguiente: $n \rightarrow p+e^{-}+\bar{\nu}$, en la que el último término representa la diferencia necesaria para balancear la masa-energía. Sin embargo, esa diferencia indica una antipartícula (una partícula con un estado de energía negativo): el antineutrino.

${ }^{30}$ El spin de una partícula subatómica indica la cantidad de momento angular intrínseco, es decir, aparte del momento angular que posea por estar en movimiento alrededor de algún punto.

${ }^{31}$ Véase la nota 28. 
y aunque no se conocía todavía la estructura o componentes del núcleo atómico, sugirió que debía haber partículas, de un tipo no conocido hasta el momento, que fueran las responsables de esa diferencia de energía en estas reacciones de decaimiento beta. El mismo Pauli era consciente de lo difícil que sería aceptar su conjetura; pero creía que debían explorarse todos los caminos que pudieran recuperar el principio de conservación amenazado. ${ }^{32}$ Más aún, en sus propias palabras, que denotan su preocupación epistemológica enfrentada con su esperanza científica, confiesa: "He hecho una cosa terrible. He postulado una partícula que no puede ser detectada." 33

Mientras tanto, Niels Bohr estaba preparado para abandonar el principio de conservación de la energía a nivel subatómico. ${ }^{34}$ Esto muestra la otra opción: que de no aceptarse una propuesta como la de Pauli, la teoría (el principio de conservación) debía ser abandonada.

Las evidencias independientes acerca de la existencia de los neutrinos se hizo esperar hasta la década de $1950 .^{35}$

${ }^{32}$ Pauli llamó a estas partículas "neutrones" y Enrico Fermi les dio el nombre de "neutrinos" luego de que James Chadwick, en 1932, descubriera las partículas constitutivas del núcleo atómico que hoy conocemos como "neutrones". Pauli sostiene: "Admito que mi recurso puede parecer bastante improbable desde el comienzo, porque de existir los neutrones [neutrinos, en la terminología corregida por Fermi] habrían sido descubiertos desde hace mucho. Sin embargo, el que no arriesga no gana [...]. Debemos entonces estar discutiendo seriamente cada camino a la salvación" (las cursivas y la traducción son nuestras) citado en Reines 1996, p. 317 (conferencia pronunciada cuando recibió el premio Nobel de Física en 1995 por su contribución a la detección del neutrino). Notemos hasta qué punto Pauli era consciente de que su maniobra obedecía a conservar el principio de conservación a costa de la ontología presupuesta hasta el momento por esas mismas teorías.

${ }^{33}$ Ibid., p. 318 (la traducción es nuestra). Esta afirmación está relacionada con lo baja que es la probabilidad de que un neutrino interactúe con un protón. En el trabajo citado se estima, sobre la base de los cálculos de Fermi, que si un neutrino ingresara en una cubeta de hidrógeno líquido viajaría una distancia equivalente a la que recorre la luz en mil años sin chocar con ningún núcleo de hidrógeno.

${ }^{34}$ Ibid., p. 317.

${ }^{35}$ De acuerdo con la teoría de Fermi y Pauli, el neutrino sería capaz de integrar decaimientos beta inversos: un decaimiento en el que el núcleo atómico 
El cambio ontológico involucrado en el caso que hemos descrito (de la postulación y posterior esfuerzo por detectar los neutrinos) corresponde a un cambio en el tipo de las entidades admitidas por la teoría, pero compatible con ella ya que justamente se realiza para seguir manteniendo el principio de conservación de la energía (y del momento angular intrínseco).

En este caso, la introducción del nuevo tipo de partículas, el neutrino, obligó a cambiar la taxonomía de partículas admitida por la teoría, mientras que en el caso de Neptuno, no se inaugura una nueva tipología de la clase de los planetas, y por lo tanto, no hay que variar la taxonomía.

Un ejemplo del segundo modo de modificación lo encontramos en los trabajos de Niels Bohr para resolver una incongruencia entre su modelo atómico y las leyes del electromagnetismo. Podríamos resumir así el problema planteado: si, tal como sostenían los modelos atómicos de esa época, los electrones se mueven orbitando el núcleo del átomo, ${ }^{36}$ y dado que toda carga acelerada emite radiación electromagnética, con lo cual pierde energía, entonces el electrón perdería energía paulatinamente y por lo tanto debería caer hacia el núcleo por la atracción eléctrica.

El problema era que esto no ocurría. La solución que propuso Bohr fue que las cargas en movimiento emiten, salvo en el caso del electrón en el átomo en órbitas estables. Pero si admitía esto debía entonces explicar por qué en ciertas ocasiones sí emite. Para esto propuso que en el átomo existen "niveles de energía" (que podríamos asociar con órbitas posibles en las que

interactúa con un neutrino y emite un positrón (antielectrón). Frederick Reines y Martin L. Pearl (ambos compartieron el Premio Nobel por la detección del neutrino) se centraron en el decaimiento beta del protón al interactuar con un neutrino, reacción descrita por la ecuación: $\nu+p \rightarrow n+e^{+}$. En ese decaimiento, el postitrón $e^{+}$podía ser detectado y esto daría una pista independiente (independiente del decaimiento beta habitual del que había surgido la conjetura, aunque, como se puede apreciar, de ningún modo independiente de la red teórica que se pretendía seguir sosteniendo) de la existencia de neutrinos. Véase Reines 1996, p. 318 y ss.

${ }^{36}$ Para que el electrón orbite al núcleo será necesaria una fuerza que lo mantenga ligado y, por lo tanto, habrá una aceleración centrípeta; en consecuencia, sería un caso de una carga acelerada. 
el electrón es estable), de tal manera que cuando el electrón está en uno de ellos no emite ni absorbe energía. Cuando es perturbado por algún medio y cambia de nivel, emite energía si pasa de un nivel mayor a otro menor, y absorbe en el caso en que pasa de uno menor a otro mayor.

Si analizamos el caso de Bohr desde nuestra propuesta, podremos ver que la decisión en este caso fue aceptar la falsación de la teoría, ${ }^{37}$ que sostenía que el electrón en el átomo, al ser una carga acelerada, emite; y proponer una modificación: mientras el electrón se encuentra en órbitas estables no emite ni absorbe, y sólo lo hace cuando pasa de una a otra.

Con esto no queremos decir que Bohr haya revisado todos los factores de la serie de los tipos $F$ antes de aceptar la falsación de la teoría. Recordemos que nuestra propuesta no establece reglas metodológicas, sino una justificación de la falsación en principio, de las teorías científicas junto con la ontología presupuesta. Bohr actuó como si no existiera ningún factor de la serie de los $F$ o de los $D$ y, por lo tanto, decidió considerar falsada la teoría.

Es interesante notar que la hipótesis propuesta por Bohr resulta una excepción de una ley aceptada anteriormente. ${ }^{38}$

Finalmente se puede presentar otro tipo de cambio que no necesariamente está ligado a un nuevo tipo de factor o a un factor conocido. La persistente falla predictiva lleva a un cambio de marco conceptual sin que se recurra a salvedades.

Podría mencionarse, como un caso típico de estos cambios, el paso de la mecánica clásica a la relativista, pero con esto no intentamos sostener que los motivos que llevaron a Einstein a proponer su teoría estuvieran relacionadas con las fallas predictivas que se habían establecido como resultados recalcitrantes para finales del siglo XIX. Sin embargo sí queremos destacar que el cambio de marco teórico (sin aludir a ningún tipo de

${ }^{37} \mathrm{Al}$ igual que lo intentaba frente a Pauli en cuanto a la postulación de los neutrinos.

${ }^{38}$ Quizás este tipo de propuestas que ponen en jaque la consistencia del corpus teórico sea un terreno en el que las posturas realistas o instrumentalistas de los científicos incidan de algún modo en sus actitudes metodológicas. Señalamos esta posible incidencia, aunque su análisis excede el marco del presente trabajo. 
proceso o entidad responsable de la falla predictiva) dio como resultado la desaparición de lo que hasta el momento parecía ser una anomalía. En el sentido kuhniano, la anomalía fue disuelta más que resuelta.

Podemos entonces establecer una taxonomía de cambio en ciencia ordenada según la magnitud de tal cambio:

Cambio 1: resolución de un enigma del primer tipo de los que hemos presentado. Es el caso que hemos referido como progreso conservador; por ejemplo, encontrar la descripción de un péndulo de acuerdo con las ecuaciones de la mecánica.

Este tipo de cambio no involucra ninguna novedad inesperada, y no altera la clasificación de entidades y procesos que, según nuestras mejores teorías vigentes, pueblan el mundo. Claramente es un cambio acumulativo del conocimiento en el sentido de que resuelve un problema de interés, y en cuya solución se hacen explícitos ciertos conocimientos que se encontraban en el seno de los principios teóricos admitidos (tal sería el caso de derivar las ecuaciones adecuadas para un caso contemplado por la teoría).

Cambio 2: resolución de un enigma que involucra una novedad en tanto hay una nueva entidad de un tipo conocido (de la serie $F$ ). Por ejemplo, la resolución del movimiento de Urano asociada al descubrimiento de Neptuno.

En el mismo sentido que el cambio 1, en estos cambios no se presentan novedades inesperadas, aun cuando los casos históricos puedan haber causado impacto en cuanto a la efectividad de la ciencia y haber dado la impresión de que se trataba de un descubrimiento de gran importancia. El ejemplo analizado de cambio 2 (el descubrimiento de Neptuno) muestra que más allá de su impacto como noticia, no hay nada que indique que ese descubrimiento marca una modificación cualitativa del conocimiento. No se abandona (al igual que en el cambio 1) la clasificación de cuáles son los tipos de objetos y procesos que pueblan el mundo, y tampoco se modifica nada respecto a las leyes que rigen esos procesos. Sigue siendo un cambio acumulativo en el conocimiento, aun a pesar de tener la apariencia de 
producir un avance sustancial de la disciplina. En realidad, este avance no sólo consiste en el descubrimiento —nimio - de la existencia de un particular más de un tipo de objetos conocido, sino del mismo proceso de haber surgido como consecuencia de querer salvaguardar la teoría de la amenaza de la falla predictiva. Es en la génesis de la conjetura donde radica el alto impacto del descubrimiento. ${ }^{39}$

Cambio 3: resolución de una anomalía (de acuerdo con nuestra caracterización) que involucra un cambio en la ontología presupuesta (serie de los tipos de factores $D$ ), pero no en las leyes del paradigma (caso del neutrino).

Aquí el impacto del descubrimiento es mayor que en los cambios anteriores. Se produce un cambio claramente cualitativo en el conocimiento, ya que es necesario suponer la presencia de un nuevo tipo de objetos que pueblan el mundo, y, por supuesto, esto conlleva también la necesidad de aceptar procesos nuevos; sin embargo, nada de esto amenaza en absoluto las teorías vigentes. Es una situación muy interesante en el sentido de que la ontología presupuesta por las teorías se modifica para su salvaguarda, pero mantiene la totalidad de las leyes previamente sustentadas. La falla predictiva ha sido transformada en una pista para la investigación, de modo que indica el rumbo que se ha de seguir a la vez que provee, en caso de éxito, un impacto muy significativo respecto a la confianza en la teoría. ${ }^{40}$ El ejemplo analizado (acerca del neutrino) muestra además la posibilidad de mantener durante un tiempo prolongado ${ }^{41}$ la dis-

${ }^{39}$ Hemos elegido presentar el ejemplo del descubrimiento de Neptuno que tardó unos cuantos años, pero podemos asociar con esta misma categoría de cambio 2 la postulación y búsqueda de las inhomogeneidades en la radiación cósmica de fondo. Este último descubrimiento puede asociarse con las observaciones del satélite COBE (Cosmic Background Explorer) realizadas en 1992, que confirmaron la conjetura lanzada en la segunda mitad de la década de 1960. Esto muestra que puede haber un lapso prolongado en la resolución de la situación.

${ }^{40} \mathrm{Al}$ mencionar esto no estamos sugiriendo ningún tipo de análisis sobre la medida en que estos descubrimientos contribuyen a la credibilidad asignada a la teoría.

${ }^{41} \mathrm{Al}$ igual que lo que hemos señalado en la nota 39. 
yuntiva sobre si existe un nuevo tipo de entidades o si la teoría debe ser abandonada.

Cambio 4: resolución de una anomalía que involucra un cambio ontológico y un cambio en las leyes del paradigma. En este caso, la raíz del cambio puede provenir de la introducción de un factor de la serie de los tipos $D$, no compatible con las leyes del paradigma, que lleva a un cambio de marco conceptual.

Estos cambios también son cualitativos como los de la categoría anterior en cuanto a que aceptan procesos o entidades que hasta el momento no habían sido postulados por las teorías vigentes; pero son además cambios cualitativos en un segundo sentido: las leyes vigentes luego del cambio no son las mismas que las que parecen regir después de éste. Esta categoría de cambio modifica la ontología sugerida por las teorías hasta el momento vigentes, pero también modifica las leyes y por lo tanto cuáles son las teorías que pueden seguirse sosteniendo en la nueva ontología.

Cambio 5: resolución o disolución de alguna anomalía al producirse un cambio de marco conceptual sin que haya referencia específica a factores nuevos.

En estos casos es notable la ruptura tanto en las leyes como en la clasificación, pero en el sentido de que los procesos y entidades que pueblan el mundo pasan a ser descritos, definidos y comprendidos de una manera diferente. De este modo, tales cambios parecen ser de la magnitud más grande en esta escala.

La numeración de los cambios sigue un orden creciente en su magnitud. Hablar sólo de cambio revolucionario, o cambio normal, que son los posibles tipos de cambio teóricos según Kuhn, no reflejaría todas las posibilidades que pueden ocurrir. No dudaríamos en decir que el cambio 1 no es revolucionario mientras que sí lo son los cambios 4 y 5 . Empero, la decisión de incluir en una u otra clase al cambio 3 podría ser motivo de discrepancias, puesto que en este caso no es necesario un reajuste en las leyes de la teoría para dar cuenta de los factores $D$ identificados al solucionar una anomalía, razón por la cual 
el alcance de esta modificación no tiene la magnitud de una revolución científica.

\section{Conclusiones}

Una vez producida la falla predictiva, el tipo de factores que se proponga para dar cuenta de la misma puede ser relevante para decidir si se ha producido un cambio revolucionario o simplemente uno en ciencia normal.

Si el factor propuesto es de la serie de tipos $F$ (se refiere a entidades reconocidas por el paradigma), entonces, el enigma efectivamente resuelto constituye un cambio en ciencia normal sin que sea necesario el ajuste en la clasificación ontológica reflejada por ese paradigma. Pero dado que la serie de los tipos de factores $F$ que se deben revisar en caso de estar frente a una falla predictiva es finita, resulta racional proponer un factor de la serie de tipos $D$ una vez que han fracasado los intentos de solucionar la anomalía por medio de algún factor $F$; y, por lo tanto, es posible explicar el cambio de manera racional una vez que se ha agotado esa vía de solución.

La gradación existente desde los cambios menos importantes hacia los más revolucionarios puede establecerse sobre la base del tipo de factores intervinientes en el esquema deductivo. Los cambios mínimos están asociados a la búsqueda de las condiciones iniciales y de contorno que, sumadas a las leyes, explican un determinado proceso. A este tipo de cambio le siguen los cambios no revolucionarios, en los que se descubre un nuevo particular de un tipo conocido que interviene en la explicación del fenómeno. En un grado más drástico de cambio, están los casos de descubrimiento de factores de un tipo desconocido hasta el momento, pero cuyo comportamiento es compatible con las leyes del marco teórico previo. Finalmente, en el grado más revolucionario de cambio, encontramos los dos últimos casos: uno en el que el descubrimiento de nuevos tipos de factores conlleva un cambio de marco conceptual, y otro en el que, sin hacer referencia a nuevos factores, se produce el cambio conceptual como reordenamiento de los datos explicados por un conjunto diferente de leyes. 


\section{BIBLIOGRAFÍA}

Gärdenfors, P., 1988, Knowledge in Flux. Modeling the Dynamics of Epistemic States, MIT Press, Cambridge, Mass.

Hempel, C.G., 1988, "Provisos: A Problem Concerning the Inferential Function of Scientific Theories", en A. Grünbaum y W. Salmon (comps.), The Limitations of Deductivism, University of California Press, Berkeley, pp. 19-36.

Hoyningen-Huene, P., 1993, Reconstructing Scientific Revolutions. Thomas S. Kuhn's Philosophy of Science, The University of Chicago Press, Chicago.

Kitcher, P., 1993, The Advancement of Science. Science without Legend, Objectivity without Illusions, Oxford University Press, Nueva York. [Versión en castellano: El avance de la ciencia, trads. Hector Islas y Laura Manríquez, Instituto de Investigaciones Filosóficas-UnAM, México, 2001.]

Kuhn, T.S., 1969, "Posdata: 1969”, La estructura de las revoluciones científicas, pp. 268-319.

1962, La estructura de las revoluciones científicas, trad. Agustín Contin, Fondo de Cultura Económica, México.

Miguel, H., J. Paruelo, y G. Pissinis, 2001, "La precisión y las anomalías en el cambio de teorías", en R. Caracciolo y D. Letzen (comps.), Epistemología e historia de la ciencia. Selección de trabajos de las XI Jornadas, vol. 7, no. 7, Universidad Nacional de Córdoba, Córdoba, pp. 348-356.

2000, "Gärdenfors, creencias remanentes y la operación de revisión", en P. García, S. Menna y V. Rodríguez (comps.), Epistemología e historia de la ciencia. Selección de trabajos de las X Jornadas, vol. 6, no. 6, Universidad Nacional de Córdoba, Córdoba, pp. 292-298.

, 1999, "Gärdenfors, el arraigo de creencias y el cambio teórico", en E. Sota y L. Urtubey (comps.), Epistemología e historia de la ciencia. Selección de trabajos de las IX Jornadas, vol. 5, no. 5, Universidad Nacional de Córdoba, Córdoba, pp. 304-310.

__ 1997, "Las salvedades en el cambio de teorías", en P. Morey y J. Ahumada (comps.), Epistemología e historia de la ciencia. Selección de trabajos de las VII Jornadas, Universidad Nacional de Córdoba, Córdoba, pp. 247-256.

Pérez Ransanz, A.R., 1999, Kuhn y el cambio científico, Fondo de Cultura Económica, México. 
Putnam, H., 1974, “The 'Corroboration' of Theories”, en P.A. Schilpp (comp.), The Philosophy of Karl Popper, Open Court, La Salle, Illinois, pp. 221-240.

Reines, F., 1996, "The Neutrino: From Poltergeist to Particle", Reviews of Modern Physics, vol. 68, no. 2, pp. 317-327.

Recibido el 8 de abril de 2002; revisado el 17 de septiembre de 2002; aceptado el 23 de septiembre de 2002 